\title{
Conjugation-specific small RNAs in Tetrahymena have predicted properties of scan (scn) RNAs involved in genome rearrangement
}

Kazufumi Mochizuki and Martin A. Gorovsky ${ }^{1}$

Department of Biology, University of Rochester, Rochester, New York 14627, USA

We proposed a scan-RNA model for genome rearrangement based on finding small RNAs that hybridized preferentially to micronuclear-specific sequences and on the properties of Twi1p, a PPD protein required for both sequence elimination and small RNA accumulation in Tetrahymena. Here we show that Twilp interacts with the small RNAs in both the old and the developing macronucleus, and is required for their stability. We show that the specificity of the small RNAs for micronuclearlimited sequences increases during conjugation. These results indicate that the small RNAs observed in conjugating cells have the properties predicted for scan RNAs.

Received May 10, 2004; revised version accepted June 22, 2004.

Like most ciliated protozoans, Tetrahymena thermophila (referred to as Tetrahymena below) have two structurally and functionally different nuclei in a single cell (see Karrer 2000). The diploid, germ-line micronucleus and the polyploid, somatic macronucleus are derived from the same zygotic nucleus formed by fertilization of two micronucleus-derived, haploid, meiotic nuclei during the sexual process of conjugation. Concomitant with formation of a new macronucleus during conjugation, the old macronucleus is destroyed. Most, if not all, transcription required for vegetative cell growth occurs in the macronucleus.

Tetrahymena undergoes extensive programmed genome rearrangement during conjugation (see Yao et al. 2002 ) resulting in elimination of $\sim 15 \%$ of the genome. About 6000 internal eliminated sequences (IESs), varying from 0.5 to $>20 \mathrm{~kb}$ in length, are eliminated, and flanking, macronucleus-destined sequences are ligated during macronuclear development in Tetrahymena. The precise ends of IESs can occur reproducibly at a specific site or at a limited number of alternative sites. Because no obvious consensus sequence had been found in and around IESs, it was not clear how IESs were precisely recognized until recently. We and others showed that an RNAi-related mechanism was involved in the IES elimination in Tetrahymena (Mochizuki et al. 2002; Yao et al. 2003). A

[Keywords: Tetrahymena; genome rearrangement; RNAi; PPD protein; small RNA]

${ }^{1}$ Corresponding author.

E-MAIL goro@mail.rochester.edu; FAX (585) 275-2070.

Article published online ahead of print. Article and publication date are at http://www.genesdev.org/cgi/doi/10.1101/gad.1219904.
PPD (PAZ-Piwi Domain) protein, Twilp, was required for IES elimination, and for accumulation of the siRNAlike small RNAs homologous to micronuclear-specific (largely IES) sequences. Twilp was detected only during conjugation and accumulated first in the cytoplasm, then in parental (old) macronuclei and then relocalized to developing (new) macronuclei.

Based on the results above, on the presence of micronuclear transcripts from both strands of the IESs in conjugating cells (Chalker and Yao 2001), and on the demonstration that sequences in the old macronucleus could epigenetically affect IES elimination (Chalker and Yao 1996), we proposed a model to explain how IESs, lacking any consensus sequences, are recognized during macronuclear development (Mochizuki et al. 2002; Mochizuki and Gorovsky 2004). First, the micronuclear genome is transcribed bidirectionally to make double-stranded (ds) RNAs that are processed to small RNAs by a Dicer-related RNase. We named these hypothetical small RNAs scan (scn) RNAs. We proposed that the scnRNAs are localized first to the old macronucleus, along with Twilp, and that the scnRNAs homologous to any DNA found there are degraded. We hypothesized that the remaining (i.e., micronucleus-specific) scnRNAs, still complexed with Twilp, are then transferred to the new macronucleus, where they promote elimination of the IES sequences to which they are homologous.

Here we test three critical predictions of the scnRNA hypothesis: (1) that the small RNAs are associated with Twilp; (2) that the small RNAs are transferred from old to new macronuclei; and (3) that macronuclear-specific sequences are eliminated from the small RNA population as conjugation proceeds. We show that a significant fraction of the $\sim 28-n t$ RNA can be coimmunoprecipitated with Twilp when this PPD protein is localized in either the old or the new macronucleus. Thus, much of the small RNAs is associated with Twilp and probably is transferred with it from the old to the new macronucleus. We also show that small RNAs present early in conjugation are much less specific for micronuclear DNA than for macronuclear DNA, suggesting that the enrichment for IES sequences predicted by the scnRNA hypothesis actually occurs. These results argue strongly that the small, $\sim 28-n t$ RNAs specifically expressed during conjugation have the predicted functions of the hypothetical scnRNAs.

\section{Results and Discussion}

\section{Expression of small RNA is greatly reduced} but not eliminated in TWI1 knockout cells

Previously we reported that small RNAs specifically expressed during conjugation in wild-type cells could not be detected in TWI1 (somatic) knockout cells in which all somatic (macronuclear) copies of TWI1 were replaced with a drug resistance marker (Mochizuki et al. 2002). However, low and asynchronous mating of these TWI1 somatic knockout cells prevented us from determining whether the small RNAs were absent or just greatly reduced in the absence of TWI1. Although the cause of this low and asynchronous mating was not clear, we suspected that senescence (Nanney 1974) or cellular damage 
occurred during the long period $(\sim 40 \mathrm{~d}=300 \sim 400$ generations) of culture and treatment with high concentration of the drug (up to $50 \mathrm{mg} / \mathrm{mL}$ of paromomycin sulfate) required for phenotypic assortment to produce somatic knockouts. To avoid these problems, we used a different strategy to make TWI1 knockout cells. One of the two copies of the TWI1 genes in the diploid micronucleus was replaced by the drug resistance marker and homozygous micronuclear knockout strains were made by uniparental pronuclear transfer during conjugation (Hai et al. 2000). Cells created by this process are homozygous TWI1 germ-line knockout heterokaryons, homozygous for disrupted TWI1 genes in their micronucleus and containing wild-type TWI1 genes in their macronucleus (see Fig. 1A). Two homozygous heterokaryon strains were crossed and homozygous TWI1 knockout homokaryon progeny cells, referred to as $\Delta T W I 1$ cells were obtained. These cells contained disrupted TWI1 genes in both macro- and micronuclei and have not been subjected to long periods of drug selection.

Complete replacement of TWI1 loci with the drug resistance markers was confirmed by PCR analyses (Fig. 1A). In contrast to the original somatic knockout strains, two $\triangle T W I 1$ strains mated almost normally and without delay (data not shown) and expressed the conjugation-specific PDD1 gene normally (see Fig. 1C). Thus, the low and asynchronous matings of TWI1 somatic knockout strains were not due to the absence of TWI1. As seen for somatic TWI1 knockouts (Mochizuki et al. 2002), when two $\Delta T W I 1$ strains were mated, no progeny grew upon refeeding of $>10$ million progeny tested (see Materials and methods). Again, like TWI1 somatic knockout cells, elimination of the M-region IES (Austerberry and Yao 1987) in the progeny of the $\Delta T W I 1$ strains also failed to occur (Fig. 1B), confirming that TWI1 is required for genome rearrangement.

Next, total RNA was extracted from mating wild-type or $\Delta T W I 1$ cells at different stages of conjugation and analyzed by acrylamide-urea gel electrophoresis (Fig. 1C). The small RNAs could be detected in $\Delta T W I 1$ cells although the amount was greatly reduced compared with wild-type cells. In early conjugation $(4 \mathrm{~h})$, the amount of the small RNA in TWI1 knockout cells was about one-fourth of that of wild-type cells but these RNAs did not persist into mid- to late stages of conjugation (Fig. 1C). These results suggest that TWI1 is dispensable for production of the small RNAs but is required for their stability. This view is supported by two additional observations. First, a Dicer-like protein (Dcllp), required for small RNA production, is localized in the meiotic micronucleus (K. Mochizuki and M.A. Gorovsky, unpubl.) but localization of Twilp in the micronucleus was not observed (Mochizuki et al. 2002). Second, Twilp and the small RNAs are

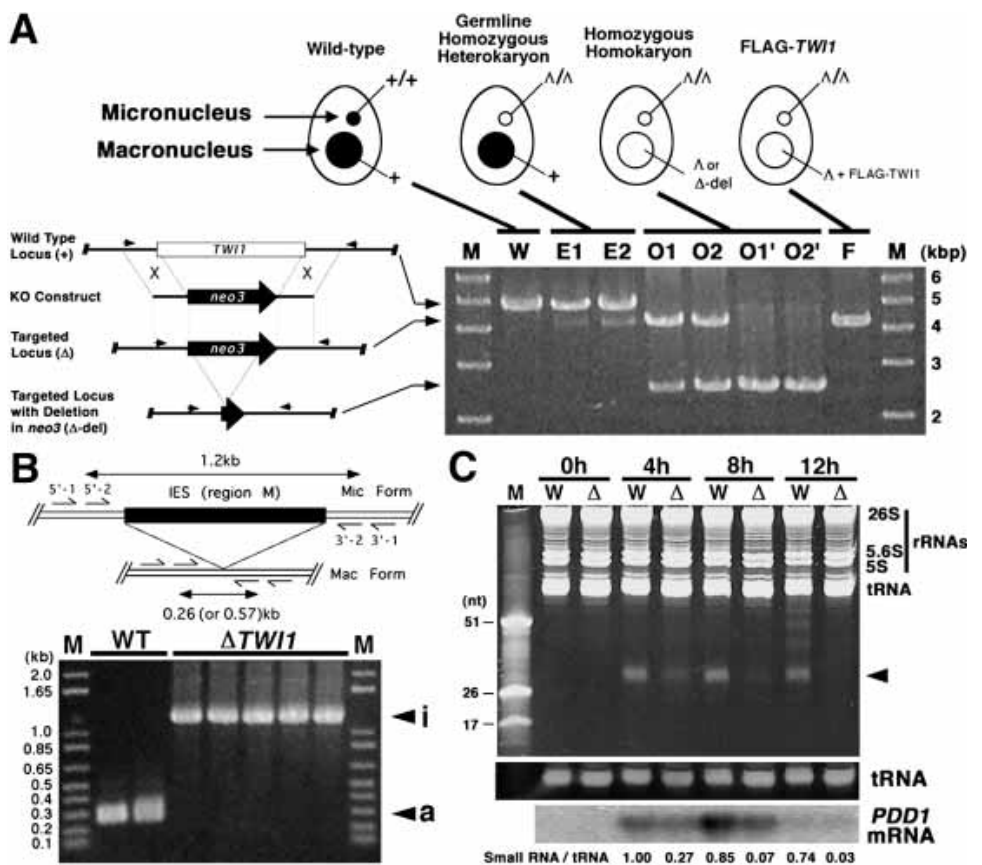

Figure 1. Construction and characterization of TWI1 germ-line knockout strains and Flag-TWI1 strains. (A) Genotyping of TWI1 germ-line knockout strains and Flag-TWI1 strains. The genotypes of the TWI1 loci in macro- and micronuclei of each cell type are illustrated at the top. TWI1 and its targeted loci are schematically drawn on the left. Arrows indicate the locations of primers used for genotyping PCR. (Lanes O1,O2) Some homozygous TWI1 knockout homokaryon strains had deletions in the neo3 cassette and gave both intact $(4.3 \mathrm{~kb})$ and deleted $(\sim 2.5 \mathrm{~kb})$ products. (Lanes $\left.\mathrm{O}^{\prime}, \mathrm{O}^{\prime}\right)$ Because macronuclear chromosomes are randomly segregated when macronuclei divide, intact targeted loci can be sorted out and knockout cells containing only deleted targeted loci can be obtained. (Lane F) The Flag-TWI1 strain was constructed in a homozygous TWI1 knockout homokaryon background and complete disruption of endogenous TWI1 loci in this strain was confirmed. Note that the region corresponding to the downstream primer was absent in pD5H8-Flag-TWI1, and thus the Flag-TWI1 gene could not be detected by PCR. (B) IES elimination assay. Double horizontal lines indicate DNA retained in the macronucleus and the filled box indicates an IES. Four primers (arrows on the horizontal lines) were used for nested PCR. The sizes of the processed (macronuclear form) and unprocessed (micronuclear form) of the M region are marked by arrowheads with " $\mathrm{a}$ " and " $\mathrm{i}$ ", respectively. (M) Molecular weight marker. (C) Expression of small RNA in wild-type and $\Delta T W I 1$ homozygous homokaryon cells. Total RNA was extracted from mating wild-type or $\triangle T W I 1$ homozygous homokaryon cells at $0,4,8$, and 12 $\mathrm{h}$ postmixing and the RNA corresponding to $5 \times 10^{4}$ cells was analyzed. The RNA was fractionated in $12 \%$ acrylamide-urea gels and stained with ethidium bromide. In vitro transcribed, 17-, 26-, and 51-nt RNAs were used as markers $(\mathrm{M})$. The gel was partially destained and tRNAs amounts were observed to normalize the loading. The amount of the small RNAs in wildtype cells at $4 \mathrm{~h}$ postmixing was adjusted as 1.00 and the relative ratio of the small RNAs at other times is indicated at the bottom. Total RNA was used for Northern hybridization with a probe for PDD1 (631-943 bp, GenBank TTU66363) mRNA which is specifically expressed in conjugating cells. associated with each other in early to late stages of conjugation (see Fig. 2). These results indicate that formation of the small RNAs in the micronucleus precedes and is independent of its association with Twilp. Because IES elimination was not observed in the progeny of TWI1 germ-line knockout cells (Fig. 1B), it is likely that 


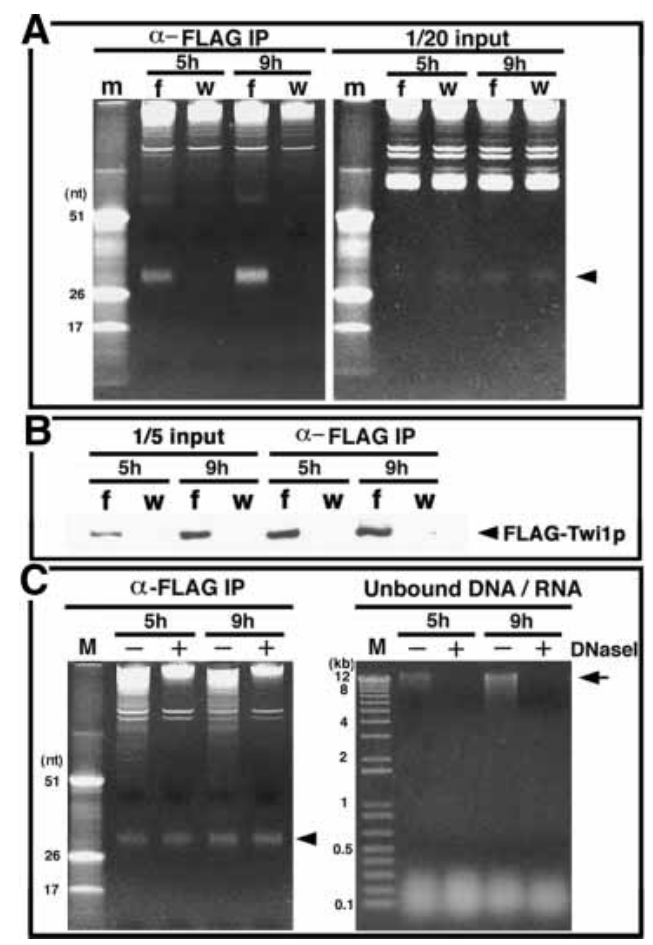

Figure 2. Coimmunoprecipitation of small RNAs with FlagTwilp. (A) Whole cell lysates were prepared from the mating of Flag-TWI1 $\times$ CU428 (f) or B2086 $\times$ CU428 (w) and the FlagTwilp-containing complex was immunoprecipitated using anti-Flag antibody. RNA extracted from one-twentieth of the lysates (right panel) and from the fraction eluted from the immunoprecipitate with Flag peptide (left panel) was analyzed by $12 \%$ acrylamide-urea gel electrophoresis followed by ethidium bromide staining. The marker $(\mathrm{m})$ was the same as in Figure 1. The arrowhead shows the position of the small RNAs. (B) FlagTwilp in the elution (right) and the lysate (left, corresponding to one-fifth of total protein used for immunoprecipitation), was analyzed on Western blot using anti-Flag antibody. (C, Left) Immunoprecipitation was performed with (+) or without (-) DNase treatment and RNA was analyzed. The arrowhead shows the position of the small RNA. (Right) To confirm that genomic DNA in lysates had been digested, nucleic acids were extracted from the unbound fraction after immunoprecipitation with phenol/chloroform and fractionated in a $1 \%$ agarose gel followed by ethidium bromide staining. The arrow indicates the position of high-molecular-weight (genomic) DNA.

either the amount of the small RNAs detected in $\Delta T W I 1$ cells is insufficient to function in genome rearrangement or that the small RNAs can not act in genome rearrangement without Twilp.

\section{Twilp interacts with small RNA}

Previously, we proposed that the small RNAs travel from old to new macronuclei in association with Twilp (Mochizuki et al. 2002). However, there was no direct evidence for the association of the small RNAs with Twilp or for their transfer. Thus, we wished to determine if Twilp interacts with the small RNAs in old and new macronuclei. To address this, we constructed strains expressing Flag-tagged Twilp. The Flag-tag-en- coding sequence was added just after the methionine initiation codon of TWI1 and a genomic DNA sequence including the whole coding and the promoter region driving TWI1 transcription was introduced into the transformation vector, pD5H8 (Godiska et al. 1993), resulting in pD5H8-Flag-TWI1. Two homozygous TWI1 germ-line knockout heterokaryons were crossed and their progeny were transformed with pD5H8-Flag-TWI1. In the transformed progeny (referred to as Flag-TWI1 strains), all endogenous TWI1 loci were disrupted in both macro- and micronuclei (confirmed by PCR, see Fig. 1A) and Flag-TWI1 expressed from pD5H8-Flag-TWI1 was the only source of TWI1 in these strains. As shown in Table 1, Flag-TWI1 strains, as well as homozygous TWI1 germ-line knockout heterokaryon strains, produced viable progeny when mated with $\triangle T W I 1$ cells. By contrast, as described above, two $\Delta T W I 1$ strains could not produce viable progeny (Table 1). Thus, Flag-Twilp expressed from pD5H8-Flag-TWI1 can replace the essential function of Twilp during conjugation, arguing that it retains normal physical interactions with other molecules required for the function of Twilp.

Next, a Flag-TWI1 strain was crossed with a wild-type strain and a whole cell lysate was prepared from the mating cells at $5 \mathrm{~h}$ (when Twilp is localized mainly in the old macronucleus) and $9 \mathrm{~h}$ (when it is mainly in the new macronucleus) postmixing. Anti-Flag antibody was used to immunoprecipitate Flag-Twilp and molecules associated with it and the immunoprecipitated components were eluted. RNA was extracted from the eluted products and analyzed by acrylamide-urea gel electrophoresis followed by ethidium bromide staining. As a control, two wild-type strains were crossed and processed in parallel. The $\sim 28$-nt small RNA coimmunoprecipitated with Flag-Twilp both in $5 \mathrm{~h}$ and $9 \mathrm{~h}$ postmixing samples (Fig. 2A). It was estimated that $239 \%(5 \mathrm{~h})$ and $41 \%(9 \mathrm{~h})$ of the small RNA in the original lysate was coimmunoprecipitated. In contrast, when two wild-type strains were crossed, the small RNAs were not precipitated. Based on the Western blot (Fig. 2B), it was estimated that $\sim 50 \%(5 \mathrm{~h})$ and $41 \%$ (9 h) of Flag-Twilp in the original lysate were collected in the elution. Assuming that unimmunoprecipitated Flag-Twilp was also complexed with the small RNA in the same manner as the precipitated Flag-Twilp, we calculate that $78 \%(5 \mathrm{~h})$ and almost $100 \%(9 \mathrm{~h})$ of the small RNAs in the cell were complexed with Twilp. These numbers were unexpectedly high because wild-type, nontagged Twilp expressed from the wild-type partner, also should be associated with the small RNAs, should not be immunoprecipitated in this experiment. Two explanations are possible for this un-

Table 1. Viability of the progeny

\begin{tabular}{lcc}
\hline Types of mating cells & Pairs tested & Progeny \\
\hline he $\Delta$ TWII-F3-1 $\times$ he $\Delta$ TWII-C4-4-3 & 288 & 30 \\
$\Delta$ TWII-5-1 $\times$ he $\Delta$ TWII-F3-1 & 288 & 26 \\
$\Delta$ TWII-5-1 $\times \Delta$ TWII-WG7-1 & 287 & 0 \\
$\Delta$ TWII-5-1 $\times$ FLAG-TWII-18 & 288 & 35 \\
\hline
\end{tabular}

At $\sim 10 \mathrm{~h}$ postmixing, single mating pairs were placed into drops of SPP medium to allow postconjugation growth. Completion of conjugation was confirmed by testing for expression of a drug resistance marker specific either for parental macronuclei or newly developed macronuclei (see Materials and Methods). 
expected result. First, because Flag-Twilp was overexpressed from the rDNA based $\mathrm{pD} 5 \mathrm{H} 8$ vector, the majority of Twilp in mating cells should be Flag-tagged. Alternatively, Twilp-small RNA complexes could contain multiple Twilp molecules. We repeated this experiment, using mating of $\Delta T W I 1$ and Flag-TWI1 strains. In this case, all Twilp molecules should be tagged. We also ran quantitative standards to ensure that the measured signals for small RNAs and for Twilp were in the linear range of quantification. In this experiment, $\sim 27 \%(5 \mathrm{~h})$ and $51 \%(9 \mathrm{~h})$ of the small RNA and $41 \%(5 \mathrm{~h})$ and $52 \%$ (9 h) of Flag-Twilp in the original lysate was coimmunoprecipitated (data not shown). Again, if immunoprecipitated and unimmunoprecipitated Flag-Twilp are similarly complexed with the small RNA, it is estimated that $\sim 66 \%(5 \mathrm{~h})$ and $97 \%(9 \mathrm{~h})$ of the small RNAs were in the Twilp complex.

Because Twilp and the small RNAs were proposed to function in genome rearrangements controlled by DNARNA interactions (Mochizuki et al. 2002), it was possible that the association of Twilp and the small RNAs was bridged by DNA. To test this, immunoprecipitation was performed with or without DNase (Fig. 2C) treatment. Similar amounts of small RNAs were precipitated in the presence or absence of DNase, suggesting that the interaction of Twilp and the small RNAs was not mediated by DNA.

As noted, at 5 and $9 \mathrm{~h}$ postmixing, the most Twilp is present in old and new macronuclei, respectively (Mochizuki et al. 2002). Thus, a large fraction of the small RNAs is likely to be associated with Twilp, and travel with it from old to new macronuclei, as predicted.

In the experiments described here, we did not detect any proteins that interacted with Flag-Twilp by silver staining although Flag-Twilp was clearly detected (data not shown). In addition the PAZ domains of five different PPD proteins in fly and human have been shown to interact directly with RNA in vitro (Lingel et al. 2003; Song et al. 2003; Yan et al. 2003). Thus, Twilp probably interacts with the small RNA directly.

\section{Small RNAs homologous to micronucleus-specific sequences are gradually enriched as conjugation proceeds}

The scnRNA model hypothesizes that the whole micronuclear genome is transcribed bidirectionally and processed to the small RNAs, and that the small RNAs then localize to the old macronucleus where those homologous to the macronuclear DNA are degraded (Mochizuki et al. 2002). If this is correct, small RNAs homologous to micronuclear-specific sequences should be enriched as conjugation proceeds. To test this prediction, purified macro- and micronuclear DNAs were probed with radioactively labeled small RNAs purified from conjugating cells at different time points. When small RNAs isolated at $2 \mathrm{~h}$ postmixing were used as probe, hybridization to micronuclear DNA was approximately three times greater than to macronuclear DNA (Fig. 3). This ratio gradually increased to $>20$ times at 8 h postmixing, when macronuclear development starts (Fig. 3). These results argue that small RNAs homologous to micronucleusspecific DNA sequences are enriched from early to midconjugation. The observation that small RNAs isolated even at early stages ( $2 \mathrm{~h}$ postmixing) of conjugation already hybridize approximately three times more to mi-

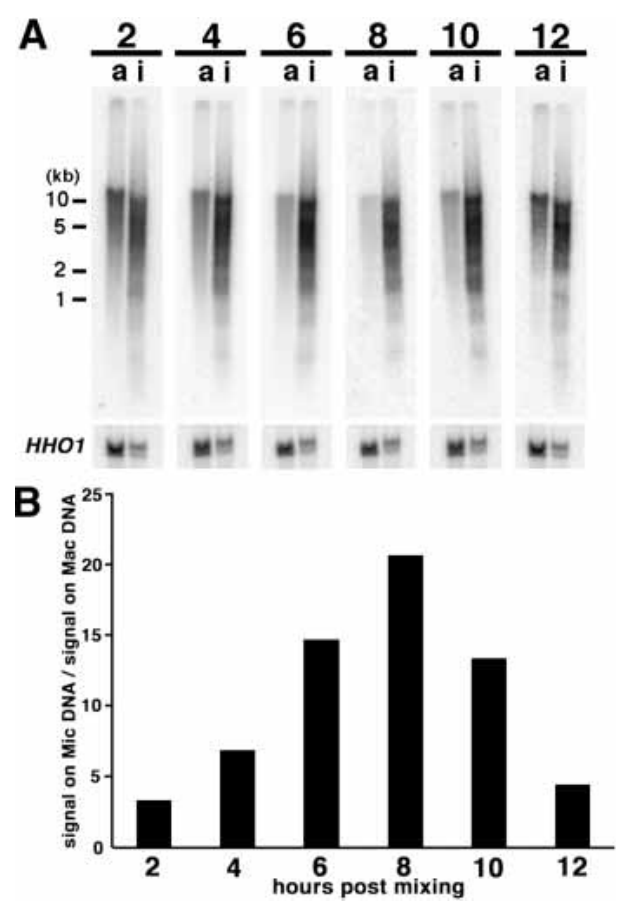

Figure 3. Hybridization of macro- and micronuclear DNA with labeled small RNAs. (A) Macronuclear (a) or micronuclear (i) DNA was isolated and digested with EcoRI. The DNA was separated in a $1 \%$ agarose gel and transferred to a membrane. To prepare the probe, total RNA from wild-type conjugating cells at $2,4,6,8,10$, and $12 \mathrm{~h}$ postmixing was fractionated in a $12 \%$ acrylamide-urea gel and the small RNA was extracted from the gel. The purified small RNA was end-labeled with ${ }^{32} \mathrm{P}$ and hybridized to macro- and micronuclear DNAs on a Southern blot. As a loading control, a probe for the HHO1 gene encoding the histone $\mathrm{H} 1$ was hybridized to the same blot. Note that approximately three times more DNA was loaded on lanes containing macronuclear DNA than on micronuclear DNA lanes. $(B)$ The ratio of the small RNAs hybridized to micronuclear DNA versus macronuclear DNA from different time points is shown as a bar graph. The loading was normalized by using the signal obtained with the HHO1 probe.

cronuclear DNA than to macronuclear DNA has four possible explantations. First, micronuclear IESs might be preferentially transcribed in early conjugation. Second, the whole micronuclear genome could be transcribed but the micronuclear sequences, many of which are repeated, might preferentially form the dsRNA substrate required for production of scnRNAs. Third, "selection" might occur so rapidly that some of small RNAs corresponding to the macronuclear sequences were already eliminated by $2 \mathrm{~h}$. Fourth, it is possible that the repeated nature of many IESs causes their transcripts to be enriched and their concentration on the blot to be high, favoring their detection. Regardless of the explanation, it is clear that the specificity of the small RNAs for micronuclear sequences increases dramatically from early to mid-conjugation. At later stages (10 and $12 \mathrm{~h}$ postmixing), the ratio decreased (Fig. 3). We speculate that small RNAs are continuously produced until late stages of conjugation (either by transcription or by amplification by RNA-dependent RNA polymerase [RdRP]) but, after new macronuclear development and old macronuclear degra- 
dation are initiated, the small RNAs cannot enter the "selection pathway" that eliminates small RNAs homologous to macronuclear sequences. At present, the detailed mechanism of "selective enrichment" of IESderived sequences is not clear. We previously proposed that a RNaseH-like activity, able to degrade RNA in RNA-DNA hybrid, may be involved in degradation of small RNAs homologous to macronucleus-destined sequences. Alternatively, because the absence of Twilp prevents accumulation of the small RNAs (Fig. 1C), it is possible that DNA-RNA pairing displaces Twilp from the small RNA-Twlip complex, allowing the small RNA to be degraded by a nonspecific RNase. Selective amplification, but not degradation, of small RNAs homologous to the micronucleus-specific DNA by RdRP could occur.

\section{The small RNAs act as scnRNAs}

In the present study, we show that Twilp is associated with 28 -nt small RNA in both old and new macronuclei (Fig. 2) and is probably required for its stability (Fig. 1C). Thus, Twilp might function as a molecular "chaperone" of the small RNAs. Because, Twilp relocalizes from old to new macronuclei (Mochizuki et al. 2002) and a large fraction of the small RNAs is associated with Twilp, the movement of the small RNAs from old to new macronuclei also is likely to occur. The increase in specificity of small RNAs for micronuclear sequences during conjugation (Fig. 3), also argues for the existence of a posttranscriptional mechanism that selects the small RNAs homologous to the micronuclear-specific sequences. These results argue that the small RNAs observed in conjugating Tetrahymena cells have functions like those hypothesized for scnRNAs (Mochizuki et al. 2002; Mochizuki and Gorovsky 2004). Thus, we propose to name the 28 -nt small RNAs specifically expressed during conjugation in Tetrahymena scnRNAs.

\section{Materials and methods}

Strains and culture conditions

Wild-type B2086 and CU428 strains of Tetrahymena thermophila (provided by Dr. P.J. Bruns, Cornell University, Ithaca, NY) were grown in SPP medium (Gorovsky et al. 1975) at $30^{\circ} \mathrm{C}$. For conjugation, logarithmically growing cells of different mating types were washed, starved $\left(16-24 \mathrm{~h}\right.$ at $\left.30^{\circ} \mathrm{C}\right)$, and mixed in $10 \mathrm{mM}$ Tris $(\mathrm{pH} 7.5)$.

Construction of TWI1 germ-line knockout

The targeting construct, with the whole TWI1 coding sequence replaced by the neo3 cassette conferring paromomycin $(\mathrm{pm})$ resistance, was described previously (Mochizuki et al. 2002). B2086 and CU428 cells were mated and the targeting construct was introduced at $2.5-3.5 \mathrm{~h}$ postmixing as described (Cassidy-Hanley et al. 1997). Two transformants that had neo3 in the TWI1 loci of both the macro- and the micronucleus were obtained. The TWI1 disrupted loci in the macronucleus were eliminated by phenotypic assortment in the absence of selection. These heterozygous transformants were crossed with "star" strain B^VII to make homozygous germ-line knockout heterokaryon strains (he $\Delta T$ TWI-C4-4-3 and hesTWI1-F3-1) by uniparental micronuclear transfer (Hai et al. 2000). Then two homozygous germ-line knockout heterokaryons were crossed to obtain homozygous homokaryon strains, referred to as $\Delta T W I 1$. Complete elimination of TWI1 genes was confirmed by PCR. In some $\Delta T W I 1$ strains, the neo3 cassette was partially eliminated from the macronucleus probably by a mechanism similar to that used to eliminate IESs (Yao et al. 2003). The remaining neo3 cassettes were eliminated by phenotypic assortment to make pm-sensitive $\Delta$ TWI1 strains ( $\Delta$ TWI1-5-1 and $\Delta$ TWI1-WG7-1). These strains can be distinguished from their progeny in the viability test and IES elimination analyses by their drug sensitivity.
Construction of Flag-TWIl strain

Flag-encoding sequence was added immediately after the initiation codon of the TWI1 gene by overlapping PCR. The primers used for the PCR were $5^{\prime}$-TWI1-NotI, 5'-CAGCGGCCGCCGATCCTTTCTCTAT GTGTCCAC-3'; Flag-TWI1-RV, 5'-CTTATCGTCGTCATCCTTGTAAT CcatGGATGTTAATTATATCGCTT-3'; Flag-TWI1-FW, 5'-GATTACA AGGATGACGACGATAAGTCTAACAAAGGCCTTGTCTA-3'; $\quad 3^{\prime}$ TWI1-NotI, 5'-CAGCGGCCGCACATAAAACGATAGCTAT-3'. NotI site is underlined, Flag-encoding sequence is in italics, and the TWI1 start codon is in lower case. 5'-TWI1 and Flag-TWI1 RV were used to amplify $\sim 800 \mathrm{bp}$ upstream from $5^{\prime}$ UTR, $5^{\prime}$ UTR, and the codon for the first methionine followed by the Flag sequence. Also, Flag-TWI1 FW and 3'-TWI1 were used to amplify the Flag-encoding sequence followed by the second amino acid coding codon, the rest of the coding sequence, $3^{\prime} \mathrm{UTR}$ and $\sim 1.1 \mathrm{kbp} 3$ ' of noncoding sequence. Then these two fragments were combined by overlapping PCR using primers $5{ }^{\prime}$-TWI1 and 3 '-TWI1. The amplified product was digested with NotI and inserted into the NotI site of the ribosomal DNA vector, pD5H8 (provided by Dr. M.C. Yao, Fred Hutchinson Cancer Research Center, Seattle, WA; Godiska et al. 1993), resulting pFlag-TWI1. pD5H8 confers pm resistance in Tetrahymane cells.

Homozygous germ-line knockout heterokaryon strains heATWI1C44-3 and he $\Delta$ TWI1F3-1 were mated and pFlag-TWI1 was introduced at 10 $\mathrm{h}$ postmixing as above. Cells were incubated in $10 \mathrm{mM}$ Tris $(\mathrm{pH} 7.5)$ for $24 \mathrm{~h}$ at $30^{\circ} \mathrm{C}$ and then refed by adding an equal amount of $2 \times$ SPP. One hundred micrograms per milliliter of pm (paromomycin sulfate, Sigma) was added $3 \mathrm{~h}$ after refeeding and cells were aliquoted to the microtiter plates. The cells were incubated until resistant clones were grown up. Although the progeny of hesTWI1C4-4-3 and hesTWI1F3-1 had pm resistance genes in the disrupted TWI1 loci, these were driven by the cadmium inducible MTT1 promoter and thus were not expressed in the absence of $\mathrm{Cd}^{2+}$ in the medium. To select cells with increasing numbers of the pFlag-TWI1-derived rDNA, the transformants were subjected to step-wise selection in increasing concentrations of pm, starting from 100 $\mu \mathrm{g} / \mathrm{mL}$ to a final of 3-4 mg/mL, above which the cells failed to grow. One of these strains $\Delta T W I 1-$ Flag-TWI1-18 was used for further experiments.

Viability test

To test the viability of the progeny of $\Delta T W I 1$ cells, starved $\Delta T W I 1-5-1$ and $\Delta$ TWI1-WG7-1 cells ( $1 \times 10^{7}$ cells each) were mixed to initiate mating and refed at $24 \mathrm{~h}$ postmixing by adding an equal amount of $2 \times$ SPP. $\mathrm{CdCl}_{2}$ was added $(0.5 \mu \mathrm{g} / \mathrm{mL}$ final) at $3 \mathrm{~h}$ after refeeding and cells were cultured for $1 \mathrm{~h}$. Then $\mathrm{CdCl}_{2}$ (to $1 \mu \mathrm{gg} / \mathrm{mL}$ total $)$ and $\mathrm{pm}(120 \mu \mathrm{g} / \mathrm{mL}$ final) were added and the culture was aliquoted to microtiter plates. Microscopic analyses indicated that $\sim 65 \%$ of the cells were exconjugants at 24 $\mathrm{h}$ postmixing. No pm resistant progeny were obtained. Thus, the viability was estimated as less than one out of $1.3 \times 10^{7}$.

To determine the functionality of Flag-Twilp, $\Delta$ TWI1-Flag-TWI1-18 and $\triangle T W I 1-5-1$ were crossed, individual pairs were placed into SPP drops at $8-10 \mathrm{~h}$ postmixing, and cultured at $30^{\circ} \mathrm{C}$. At $48-60 \mathrm{~h}$ after cloning, drops were examined for growth of cells. Then, the phenotypes of growing cells were tested to determine whether cells had completed or aborted conjugation. These cells were incubated with $120 \mu \mathrm{g} / \mathrm{ml} \mathrm{pm}$ with or without $1 \mathrm{\mu g} / \mathrm{mL} \mathrm{CdCl} 2$. Clones that showed $\mathrm{pm}$ resistance with $\mathrm{CdCl}_{2}$ but were sensitive in its absence were scored as progeny. This assay depends on the fact that $\triangle T$ TWI1-Flag-TWI1-18 had pD5H8 derived rDNA gene that conferred pm resistance in Tetrahymena without $\mathrm{CdCl}_{2}$ in the macronucleus while both $\Delta$ TWI1-Flag-TWI1-18 and $\Delta T W I 1-5-1$ had neo3 that conferred pm resistance in Tetrahymena with $\mathrm{CdCl}_{2}$ in the micronucleus. As controls, conjugations of $\Delta T W I 1-5-1 \times$ he $\Delta T W I 1-F 3-1$ or $\Delta$ TWI1-5-1 $\times \Delta$ TWI1-WG7-1 were also tested. To determine the completion of conjugation in these control experiment, cells were cultured in $1 \times \mathrm{SPP}$ including $120 \mu \mathrm{g} / \mathrm{mL} \mathrm{pm}$ and $1 \mu \mathrm{g} / \mathrm{mL} \mathrm{CdCl}_{2}$ and scored for cells resistant to $\mathrm{pm}$.

Small RNA and IES elimination analysis

Total RNA extraction and analysis of the small RNAs were performed as described (Mochizuki et al. 2002). IES elimination was analyzed as described (Mochizuki et al. 2002) except cells were analyzed at 48 h postmixing.

Coimmunoprecipitation

CU428 or $\Delta$ TWI1-WG7-1 and $\Delta$ TWI1-Flag-TWI1-18 $\left(2 \times 10^{5} \mathrm{cells} / \mathrm{mL}\right.$ of each $)$ were mated. At 5 and $9 \mathrm{~h}$ postmixing, $2 \times 10^{6}$ cells were collected and homog- 
enized in $1 \mathrm{~mL}$ lysis solution $(50 \mathrm{mM}$ Tris at $\mathrm{pH} 7.5,150 \mathrm{mM} \mathrm{NaCl}, 2 \mathrm{mM}$ $\mathrm{MgCl}_{2}, 1 \%$ Tween 20, $1 \times$ Complete Proteinase Inhibitor (Roche), $2 \mathrm{mM}$ phenyl methyl sulfonylfluoride (Roche), $0.2 \mathrm{mg} / \mathrm{mL}$ Ribonucleoside Vanadyl Complex (New England Biolabs). Insoluble materials were sedimented at 10,000 $\times \mathrm{g}$ for $15 \mathrm{~min}$ and the supernatant was used for immunoprecipitation. Anti-Flag M2agarose affinity gel (Sigma) was mixed with the supernatant to precipitate the Flag-Twilp complex. After overnight incubation at $4^{\circ} \mathrm{C}$, the gel was rinsed in wash buffer $(50 \mathrm{mM}$ Tris at $\mathrm{pH} 7.5,150 \mathrm{mM} \mathrm{NaCl}, 2 \mathrm{mM} \mathrm{MgCl}$ ) and the complex was eluted in $150 \mu \mathrm{g} / \mathrm{mL} 3 \times$ Flag peptide (Sigma) in wash buffer. Nucleic acids were extracted from the eluted fraction with phenol/chloroform and then ethanol precipitated. The small RNAs were analyzed as described (Mochizuki et al. 2002). The intensities of bands were measured using NIH Image version 1.59. For DNase treatment, $0.1 \mathrm{mg} / \mathrm{mL}$ of DNase I (RNase free, Sigma) was added to the initial lysate. After immunoprecipitation, nucleic acid was extracted from the unbound fraction and analyzed on $1 \%$ agarose gels to check DNA digestion.

Southern hybridization with labeled small RNAs

The small RNAs were purified from acrylamide urea gels and end-labeled as described (Mochizuki et al. 2002). Southern hybridization to isolated macro- and micronuclear DNA was performed as described (Mochizuki et al. 2002). To normalize the loading of DNA, the membranes were stripped and reprobed with $H H O 1$ probe (random primed DNA probe, 2.4-kb EcoRI-HindIII fragment of pTtDR1, Wu et al. 1986). The radioactivity on the blot was analyzed using a PhosphorImager (Molecular Dynamics/Amersham).

\section{Acknowledgments}

We thank Josephine Bowen for critical reading of the manuscript. This work was supported by grant GM21793 from the National Institutes of Health.

The publication costs of this article were defrayed in part by payment of page charges. This article must therefore be hereby marked "advertisement" in accordance with 18 USC section 1734 solely to indicate this fact.

\section{References}

Austerberry, C.F. and Yao, M.C. 1987. Sequence structures of two developmetally regulated, alternative DNA deletion junctions in Tetrahymena thermophila. Mol. Cell. Biol. 8: 3947-3950.

Cassidy-Hanley, D., Bowen, J., Lee, J.H., Cole, E., VerPlank, L.A., Gaertig, J., Gorovsky, M.A., and Bruns, P.J. 1997. Germline and somatic transformation of mating Tetrahymena thermophila by particle bombardment. Genetics 146: 135-147.

Chalker, D.L. and Yao, M.C. 1996. Non-Mendelian, heritable blocks to DNA rearrangement are induced by loading the somatic nucleus of Tetrahymena thermophila with germ line-limited DNA. Mol. Cell. Biol. 16: 3658-3667.

- 2001. Nongenic, bidirectional transcription precedes and may promote developmental DNA deletion in Tetrahymena thermophila. Genes \& Dev. 15: 1287-1298.

Godiska, R., James, C., and Yao, M.C. 1993. A distant 10-bp sequence specifies the boundaries of a programmed DNA deletion in Tetrahymena. Genes \& Dev. 7: 2357-2365.

Gorovsky, M.A., Yao, M.C., Keevert, J.B., and Pleger, G.L. 1975. Isolation of micro- and macronuclei of Tetrahymena pyriformis. Meth. Cell Biol. 9: 311-327.

Hai, B., Gaertig, J., and Gorovsky, M.A. 2000. Knockout heterokaryons enable facile mutagenic analysis of essential genes in Tetrahymena. Meth. Cell Biol. 62: 513-531.

Karrer, K.M. 2000. Tetrahymena genetics: Two nuclei are better than one. Meth. Cell Biol. 62: 127-186.

Lingel, A., Simon, B., Izaurralde, E., and Sattler, M. 2003. Structure and nucleic-acid binding of the Drosophila Argonaute 2 PAZ domain. Nature 426: 465-469.

Mochizuki, K. and Gorovsky, M.A. 2004. Small RNAs in genome rearrangement in Tetrahymena. Curr. Opin. Genet. Dev. 14: 181-187.

Mochizuki, K., Fine, N.A., Fujisawa, T., and Gorovsky, M.A. 2002. Analysis of a piwi-related gene implicates small RNAs in genome rearrangement in Tetrahymena. Cell 110: 689-699.
Nanney, D.L. 1974. Aging and long-term temporal regulation in ciliated protozoa. A critical review. Mech. Ageing Dev. 3: 81-105.

Song, J.J., Liu, J., Tolia, N.H., Schneiderman, J., Smith, S.K., Martienssen, R.A., Hannon, G.J., and Joshua-Tor, L. 2003. The crystal structure of the Argonaute2 PAZ domain reveals an RNA binding motif in RNAi effector complexes. Nat. Struct. Biol. 10: 1026-1032.

Wu, M., Allis, C.D., Richman, R., Cook, R.G., and Gorovsky, M.A. 1986. An intervening sequence in an unual histone H1 gene of Tetrahymena thermophila. Proc. Nat1. Acad. Sci. 83: 8674-8678.

Yan, K.S., Yan, S., Farooq, A., Han, A., Zeng, L., and Zhou, M.M. 2003. Structure and conserved RNA binding of the PAZ domain. Nature 426: $468-474$.

Yao, M.C., Duharcourt, S., and Chalker, D.L. 2002. Genome-wide rearrangements of DNA in ciliates. In Mobile DNA II (eds. N. Craig et al.), pp. 730-758. Academic Press, New York.

Yao, M.C., Fuller, P., and Xi, X. 2003. Programmed DNA deletion as an RNA-guided system of genome defense. Science 300: 1581-1584. 


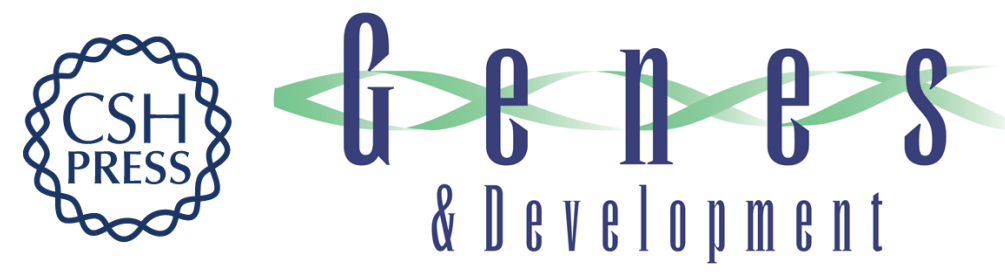

\section{Conjugation-specific small RNAs in Tetrahymena have predicted properties of scan (scn) RNAs involved in genome rearrangement}

Kazufumi Mochizuki and Martin A. Gorovsky

Genes Dev. 2004, 18:

Access the most recent version at doi:10.1101/gad.1219904

References This article cites 16 articles, 6 of which can be accessed free at:

http://genesdev.cshlp.org/content/18/17/2068.full.html\#ref-list-1

License

Email Alerting Receive free email alerts when new articles cite this article - sign up in the box at the top Service right corner of the article or click here.

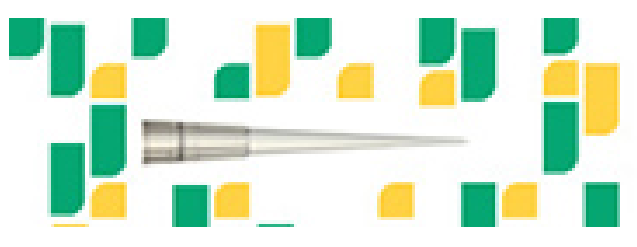

Focused on your science. 